PROCEEDINGS OF THE

AMERICAN MATHEMATICAL SOCIETY

Volume 130, Number 1, Pages 271-281

S 0002-9939(01)06381-X

Article electronically published on July 25, 2001

\title{
REAL EQUIVARIANT BORDISM AND STABLE TRANSVERSALITY OBSTRUCTIONS FOR $\mathbb{Z} / 2$
}

\author{
DEV SINHA
}

(Communicated by Ralph Cohen)

\begin{abstract}
In this paper we compute homotopical equivariant bordism for the group $\mathbb{Z} / 2$, namely $M O_{*}^{\mathbb{Z} / 2}$, geometric equivariant bordism $\mathfrak{N}_{*}^{\mathbb{Z} / 2}$, and their quotient as modules over geometric bordism. This quotient is a module of stable transversality obstructions. We construct these rings from knowledge of their localizations.
\end{abstract}

In this paper we compute homotopical equivariant bordism for the group $\mathbb{Z} / 2$, namely $M O_{*}^{\mathbb{Z} / 2}$, geometric equivariant bordism $\mathfrak{N}_{*}^{\mathbb{Z} / 2}$, and their quotient as $\mathfrak{N}_{*}^{\mathbb{Z} / 2}$ modules. In doing these computations, we use the techniques of [10. Note that $\mathfrak{N}_{*}^{\mathbb{Z} / 2}$ has been computed in 11 and $M O^{\mathbb{Z} / 2}$ was described as a two-stage Postnikov system in [5], although explicit generators and relations were not given for $M O_{*}^{\mathbb{Z} / 2}$. The results here are explicit, and hence new, making systematic use of localization. The computation of the quotient of these theories, a module of stable transversality obstructions closely related to those of [6], is new. Bröcker and Hook showed that one may obtain $M O^{\mathbb{Z} / 2}$ from $\mathfrak{N}^{\mathbb{Z} / 2}$ by introducing suspension isomorphisms for non-trivial representations 2. Our computations capture the algebra of this stabilization procedure.

The paper is structured as indicated by section titles; we give basic definitions, then statements of theorems, and finally proofs. The proofs aim to be complete, assuming the reader has no familiarity with equivariant homotopy theory. Hence, preliminary results proven are well known to experts.

Thanks go to Peter Landweber for a close reading of this paper.

\section{Definitions}

Although all of the basic constructions can be made for an arbitrary compact Lie group, we state things only for the group $\mathbb{Z} / 2$. For a thorough introduction to equivariant bordism see Chapter 15 in 8 by Costenoble. Let $\tau$ denote the trivial one-dimensional real representation of $\mathbb{Z} / 2$, and let $\sigma$ denote the non-trivial one in which the non-trivial element acts on $\mathbb{R}$ by multiplication by -1 . Let $B O^{\mathbb{Z} / 2}(n)$ be the Grassmannian of $n$-dimensional subspaces of $\mathcal{U}=\bigoplus_{\infty}(\tau \oplus \sigma)$, with $\mathbb{Z} / 2$ action inherited from $\mathcal{U}$, and let $\xi_{n}^{\mathbb{Z} / 2}$ be the universal $\mathbb{Z} / 2 n$-dimensional bundle over $B O^{\mathbb{Z} / 2}(n)$, and $T\left(\xi_{n}^{\mathbb{Z} / 2}\right)$ its associated Thom space. Given a representation $V$, let $S^{V}$ be the one-point compactification of $V$.

Received by the editors May 19, 2000.

2000 Mathematics Subject Classification. Primary 57R85.

(C)2001 American Mathematical Society 
Let $\mathfrak{N}_{*}^{\mathbb{Z} / 2}$ denote the ring of bordism classes of $\mathbb{Z} / 2$-manifolds (manifolds with an involution), where bordism is defined in the usual way using manifolds with boundary as in [4. To define homotopical bordism, first note that for any representation $V$ there are maps $S^{V} \wedge T\left(\xi_{n}^{\mathbb{Z} / 2}\right) \rightarrow T\left(\xi_{n+|V|}^{\mathbb{Z} / 2}\right)$ defined by passage to Thom spaces of the map classifying $V \times \xi_{n}^{\mathbb{Z} / 2}$. For non-negative $n, M O_{n}^{\mathbb{Z} / 2}$ is the colimit

$$
\varliminf_{\longrightarrow} W\left[S^{n \tau \oplus W}, T\left(\xi_{|W|}^{\mathbb{Z} / 2}\right)\right]^{\mathbb{Z} / 2}
$$

where $[,]^{\mathbb{Z} / 2}$ denotes the set of homotopy classes of $\mathbb{Z} / 2$-maps, which in this case of taking maps from spheres is an abelian group. As in the ordinary setting, the Pontryagin-Thom construction gives rise to a map $\mathfrak{N}_{n}^{\mathbb{Z} / 2} \rightarrow M O_{n}^{\mathbb{Z} / 2}$ (again see [4]). But we will see that this map is not an isomorphism. Indeed, we extend the definition of $M O_{n}^{\mathbb{Z} / 2}$ to negative degrees in the standard way as

$$
\lim _{W}\left[S^{W}, T\left(\xi_{|W|+|n|}^{\mathbb{Z} / 2}\right)\right]^{\mathbb{Z} / 2} \text {. }
$$

We will see that these groups are non-zero for any $n$, whereas $\mathfrak{N}_{n}^{\mathbb{Z} / 2}$ are zero for negative $n$ by definition. We may in fact define an equivariant spectrum $M O^{\mathbb{Z} / 2}$, equipped with deloopings for any formal difference of representations of $\mathbb{Z} / 2$, giving rise to associated homology and cohomology theories where $M O_{*}^{\mathbb{Z} / 2} \cong$ $M O_{*}^{\mathbb{Z} / 2}(p t.) \cong M O_{\mathbb{Z} / 2}^{-*}(p t$.). There are periodicity isomorphisms (see [8]) which imply that $M O_{V-W}^{\mathbb{Z} / 2}$ depends only on the virtual dimension of $V-W$, so we restrict our attention to integer gradings.

The difference between geometric and homotopical bordism arises from the breakdown of transversality in the presence of a group action. The most important examples of classes in $M O_{*}^{\mathbb{Z} / 2}$ not coming from geometric bordism are the Euler classes (indeed, for $\mathbb{Z} / 2$ we will see that these are essentially the only examples). The representation $\sigma$ defines a $\mathbb{Z} / 2$ vector bundle over a point by projection. There are no non-zero equivariant sections of this bundle. The Euler class $e_{\sigma} \in M O_{\mathbb{Z} / 2}^{1}(p t$.) reflects the equivariant non-triviality of this bundle. Explicitly, given a representation $V$ define the Euler class $e_{V}$ to be the class of the composite $S^{0} \subset S^{V} \rightarrow T\left(\xi_{|V|}^{\mathbb{Z} / 2}\right)$ in $M O_{-|V|}^{\mathbb{Z} / 2}$, where the second map is defined by passing to Thom spaces the inclusion of $V$ as a fiber of $\xi_{|V|}$.

\section{Statements of the theOREMS}

Euler classes play an important role at every step in this paper. Tom Dieck [7, refining ideas of Atiyah and Segal, showed that localization by inverting these Euler classes corresponds to "reduction to fixed sets". We take tom Dieck's work as a starting point, translating his results from the complex setting. Once we are taking geometric fixed sets, we can make explicit computations.

Let $\mathbb{P}(V)$ be the projective space of one-dimensional subspaces of a representation $V$, with inherited $\mathbb{Z} / 2$-action, and let $[\mathbb{P}(V)] \in M O_{|V|-1}^{\mathbb{Z} / 2}$ be the image of the bordism class of $\mathbb{P}(V)$ under the Pontryagin-Thom map. Let $R_{*}$ be the sub-algebra of $M O_{*}^{\mathbb{Z} / 2}$ generated by the Euler class $e_{\sigma}$ and $[\mathbb{P}(n \tau \oplus \sigma)]$, as $n$ ranges over natural numbers, and let $S$ be the multiplicative set in $R_{*}$ generated by $e_{\sigma}$. By abuse, use $S$ to denote the same multiplicative set in $\mathrm{MO}_{*}^{\mathbb{Z} / 2}$. 
Theorem 2.1. The canonical map $S^{-1} R_{*} \rightarrow S^{-1} M O_{*}^{\mathbb{Z} / 2}$ is an isomorphism.

In other words, any class in $M O_{*}^{\mathbb{Z} / 2}$ can be multiplied by some power of $e_{\sigma}$ to get a class in $R_{*}$ modulo the kernel of the localization map (which is in fact zero by the next theorem). Hence, to understand $M O_{*}^{\mathbb{Z} / 2}$ it suffices to understand $R_{*}$ and division by $e_{\sigma}$. We will prove Theorem 2.1 by explicit computation. It will follow from the computation of $S^{-1} M O_{*}^{\mathbb{Z} / 2}$ that $R_{*}$ is a polynomial algebra. In fact, $R_{*}$ is a maximal polynomial subalgebra of $M O_{*}^{\mathbb{Z} / 2}$.

Remarkably, divisibility by $e_{\sigma}$ is closely tied to the interplay between equivariant and ordinary bordism. Let $\alpha: M O_{*}^{\mathbb{Z} / 2} \rightarrow \mathfrak{N}_{*}$ be the augmentation map, that is, the map which forgets $\mathbb{Z} / 2$-action. Clearly $e_{\sigma}$ is in the kernel of $\alpha$. In some sense, this Euler class accounts for the entire kernel.

Theorem 2.2. The sequence $0 \rightarrow M O_{*}^{\mathbb{Z} / 2} \stackrel{\cdot e_{\sigma}}{\rightarrow} M O_{*}^{\mathbb{Z} / 2} \stackrel{\alpha}{\rightarrow} \mathfrak{N}_{*} \rightarrow 0$ is exact.

Using the exactness of this sequence, we define an operation on $M O_{*}^{\mathbb{Z} / 2}$. First note that the augmentation map has a splitting $\iota: \mathfrak{N}_{*} \rightarrow M O_{*}^{\mathbb{Z} / 2}$ which we may define by taking a geometric representative for a class, imposing a trivial action, and passing to homotopical bordism through the Pontryagin-Thom map. Hence there is also a splitting of multiplication by $e_{\sigma}$.

Definition 2.3. For any $x \in M O_{*}^{\mathbb{Z} / 2}$ define $\bar{x}$ to be $\iota \circ \alpha(x)$. Then $x-\bar{x}$ is in the kernel of $\alpha$, so we define $\Gamma(x)$ to be the unique class such that $e_{\sigma} \Gamma(x)=x-\bar{x}$.

Directly from Theorem 2.1 and Theorem 2.2 we may deduce the following.

Theorem 2.4. $M O_{*}^{\mathbb{Z} / 2}$ is generated over $\mathfrak{N}_{*}$ by $e_{\sigma}$ and classes $\Gamma^{i}([\mathbb{P}(n \tau \oplus \sigma)])$ where $i$ and $n$ range over natural numbers. Relations are

- $e_{\sigma} \Gamma(x)=x-\bar{x}$

- $\Gamma(x y)=\Gamma(x) y-\bar{x} \Gamma(y)$,

where $x$ and $y$ range over the classes $\Gamma^{i}([\mathbb{P}(n \tau \oplus \sigma)])$.

When $x$ is a geometric class, that is, $x$ is in the image of some $[M]$ under the Pontryagin-Thom map, there is a geometric construction of $\Gamma(x)$, a construction which dates back to work by Conner and Floyd 4 .

Definition 2.5. Let $\gamma(M)=M \times_{\mathbb{Z} / 2} S^{1}$, where $S^{1}=\left\{(x, y) \in \mathbb{R}^{2} \mid x^{2}+y^{2}=1\right\}$ has antipodal $\mathbb{Z} / 2$ action and $\mathbb{Z} / 2$ acts on the quotient by the rule $g \cdot[m, x, y]=$ $[m,-x, y]$, where $g$ is the non-trivial element of $\mathbb{Z} / 2$ and the brackets denote taking equivalence classes.

Theorem 2.6. $\Gamma([M])=[\gamma(M)]$.

Because we have a geometric model for this operation, and because we may for $\mathbb{Z} / 2$ identify geometric equivariant bordism as a sub-ring of homotopical equivariant bordism, we also have explicit understanding of the geometric theory.

Theorem 2.7. The geometric $\mathbb{Z} / 2$ bordism ring $\mathfrak{N}_{*}^{\mathbb{Z} / 2}$ is the sub-ring of $M O_{*}^{\mathbb{Z} / 2}$ generated over $\mathfrak{N}_{*}$ by the classes $\left[\gamma^{i}(\mathbb{P}(n \tau \oplus \sigma))\right]$.

Finally, we may identify the quotient $M O_{*}^{\mathbb{Z} / 2} / \mathfrak{N}_{*}^{\mathbb{Z} / 2}$, which represents some stable transversality obstructions, as a $\mathfrak{N}_{*}^{\mathbb{Z} / 2}$-module. 
Theorem 2.8. The $\mathfrak{N}_{*}^{\mathbb{Z} / 2}$-module $M O_{*}^{\mathbb{Z} / 2} / \mathfrak{N}_{*}^{\mathbb{Z} / 2}$ is generated by classes $x_{k}, k \in \mathbb{N}$, in degree $-k$, with relations $[\gamma(M)] x_{k}=[M] x_{k-1}-[\bar{M}] x_{k-1}$. The class $x_{k}$ is the image of the Euler class $e_{k \sigma}$ under the canonical map from $\mathrm{MO}_{*}^{\mathbb{Z} / 2}$ to this quotient.

Hence the Euler classes are essentially the only transversality obstructions in this setting. This is not true in general, as for $\mathbb{Z} / 3$ for example the class $S^{\rho} \stackrel{z \mapsto z^{2}}{\rightarrow}$ $S^{\rho^{\otimes 2}} \rightarrow T\left(\xi_{2}^{\mathbb{Z} / 3}\right)$ is a non-trivial class in the quotient of homotopical and geometric bordism which is not a multiple of an Euler class.

\section{Proofs}

As should be expected, our computations start with exact sequences due to Conner and Floyd, and tom Dieck. Indeed, the work of [1] and [5] starts from these exact sequences. Most of the results in this section which lead up to the final computation have been known to experts for some time.

3.1. The Conner-Floyd exact sequence. Our goal is to prove the following theorem, and then explicitly compute some of the terms.

Theorem 3.1 (Conner-Floyd). There are maps $\eta, \phi$ and $\delta$, to be defined below, so that the sequence

$$
\cdots \rightarrow \mathfrak{N}_{*}(B \mathbb{Z} / 2) \stackrel{\eta}{\rightarrow} \mathfrak{N}_{*}^{\mathbb{Z} / 2} \stackrel{\phi}{\rightarrow} \bigoplus_{k} \mathfrak{N}_{*-k}(B O(k)) \stackrel{\delta}{\rightarrow} \mathfrak{N}_{*-1}(B \mathbb{Z} / 2) \rightarrow \cdots
$$

is exact.

Historically, Conner and Floyd developed their exact sequence geometrically. We proceed in what is in some sense the reverse of historical order, starting with the long exact sequence in $\mathfrak{N}_{*}^{\mathbb{Z} / 2}$ theory of the based pair $\left(c(E \mathbb{Z} / 2)_{+}, E \mathbb{Z} / 2+\right)$, where $E \mathbb{Z} / 2$ is a contractible space on which $\mathbb{Z} / 2$ acts freely (for example, the unit sphere in $\left.\bigoplus_{\infty} \sigma\right)$ and $c(E \mathbb{Z} / 2)_{+}$denotes the cone on $E \mathbb{Z} / 2$ with a disjoint basepoint added. To be complete, we include the definition of relative bordism.

Definition 3.2. Let $(X, A)$ be an admissible pair of $\mathbb{Z} / 2$-spaces. A $\mathbb{Z} / 2$-manifold with reference to $(X, A)$ is a pair $(M, f)$ of a $\mathbb{Z} / 2$-manifold with boundary $M$ and a map $f: M \rightarrow X$ such that $f(\partial M) \subseteq A$. Two $\mathbb{Z} / 2$-manifolds $\left(M_{1}, f_{1}\right)$ and $\left(M_{2}, f_{2}\right)$ are bordant when there is a $\mathbb{Z} / 2$-manifold $(W, g)$ such that $M_{1} \sqcup M_{2}$ is $\mathbb{Z} / 2$ diffeomorphic to a codimension zero sub-manifold of $\partial W,\left.g\right|_{M_{1}}=f_{1},\left.g\right|_{M_{2}}=f_{2}$, and $g\left(\partial W-\left(M_{1} \sqcup M_{2}\right)\right) \subseteq A$.

Definition 3.3. Let $\mathfrak{N}_{n}^{\mathbb{Z} / 2}(X, A)$ denote the group of equivalence classes up to bordism of $\mathbb{Z} / 2$-manifolds with reference to $(X, A)$.

The functor $\mathfrak{N}_{*}^{\mathbb{Z} / 2}$ defines an equivariant homology theory. In this theory, the exact sequence of the pair $\left(c(E \mathbb{Z} / 2)_{+}, E \mathbb{Z} / 2_{+}\right)$reads

$$
\begin{aligned}
\cdots \rightarrow \widetilde{\mathfrak{N}^{\mathbb{Z} / 2}} *(E \mathbb{Z} / 2+) \stackrel{i_{*}}{\rightarrow} \mathfrak{N}_{*}^{\mathbb{Z} / 2} \stackrel{\stackrel{j_{*}}{\rightarrow}}{ } \\
\mathfrak{N}_{*}^{\mathbb{Z} / 2}(c(E \mathbb{Z} / 2), E \mathbb{Z} / 2) \stackrel{\partial}{\rightarrow} \widetilde{\mathfrak{N}^{\mathbb{Z} / 2}}{ }_{*-1}(E \mathbb{Z} / 2+) \rightarrow \cdots .
\end{aligned}
$$

We will identify the first and third terms in this exact sequence with more familiar bordism modules. Note that these identifications are standard, and are done in some generality by Costenoble in Chapter 15 of [8]. We start with analysis of the term 
$\mathfrak{N}_{*}^{\mathbb{Z} / 2}(c(E \mathbb{Z} / 2), E \mathbb{Z} / 2)$ and the map $j_{*}$. Form the bordism module of $\mathbb{Z} / 2$-manifolds with free boundary, where we define the bordism relation as in Definition 3.2

Proposition 3.4. The module $\mathfrak{N}_{*}^{\mathbb{Z} / 2}(c(E \mathbb{Z} / 2), E \mathbb{Z} / 2)$ is naturally isomorphic to the bordism module of $\mathbb{Z} / 2$-manifolds with free boundary.

Proof. Any manifold with reference to $(c(E \mathbb{Z} / 2), E \mathbb{Z} / 2)$ must have free boundary. Conversely, given a $\mathbb{Z} / 2$-manifold with free boundary, there is no obstruction to constructing a reference map to $(c(E \mathbb{Z} / 2), E \mathbb{Z} / 2)$. Applying these observations to manifolds which play the role of bordisms, we see that these correspondences are well defined up to bordism and inverse to each other.

Up to bordism, a $\mathbb{Z} / 2$-manifold with free boundary depends only on its fixed-set data.

Proposition 3.5. A $\mathbb{Z} / 2$-manifold with free boundary $M$ is bordant to any smooth neighborhood $\mathcal{N}\left(M^{\mathbb{Z} / 2}\right)$ of the fixed set of $M$ as $\mathbb{Z} / 2$-manifolds with free boundary.

Proof. Let $W=M \times[0,1]$, with "straightened angles". Then $\partial W$ is free outside of $M \times 0$ and $\mathcal{N}\left(M^{\mathbb{Z} / 2}\right) \times 1$, so $W$ is the required bordism.

Thus the map $j_{*}: \mathfrak{N}_{*}^{\mathbb{Z} / 2} \rightarrow \mathfrak{N}_{*}^{\mathbb{Z} / 2}(c(E \mathbb{Z} / 2), E \mathbb{Z} / 2)$ "reduces to fixed sets" in the sense of sending a representative of a bordism class $M$ to the bordism class of smooth neighborhoods of its fixed set. If we choose $\mathcal{N}\left(M^{\mathbb{Z} / 2}\right)$ to be a tubular neighborhood of $M^{\mathbb{Z} / 2}$, then we can use standard equivariant differential topology to identify this tubular neighborhood with a $\mathbb{Z} / 2$-vector bundle over the fixed set, where the action of $\mathbb{Z} / 2$ is free away from zero.

Proposition 3.6. $\mathfrak{N}_{*}^{\mathbb{Z} / 2}(c(E \mathbb{Z} / 2), E \mathbb{Z} / 2) \cong \bigoplus_{k} \mathfrak{N}_{*-k}(B O(k))$.

Proof. Use the identifications we have made so far to equate $\mathfrak{N}_{*}^{\mathbb{Z} / 2}(c(E \mathbb{Z} / 2), E \mathbb{Z} / 2)$ with the bordism module of $\mathbb{Z} / 2$-vector bundles over trivial $\mathbb{Z} / 2$-spaces where the action is free away from zero. The fiber of a $\mathbb{Z} / 2$-vector bundle over a trivial $\mathbb{Z} / 2$ space is a representation. Because $\mathbb{Z} / 2$ has only one non-trivial representation, the action on any fiber and thus the total space is completely determined. Hence the forgetful map from this bordism module of $\mathbb{Z} / 2$-vector bundles which are free away from the zero section to the bordism module of vector bundles is an isomorphism. The result follows from the fact that $B O(k)$ is the classifying space for vector bundles. Note that we must grade according to the dimension of the total space of the bundle in question.

Interpreting the term $\widetilde{\mathfrak{N}^{\mathbb{Z} / 2}} *(E \mathbb{Z} / 2+)$ is more immediate. Any manifold mapping to $E \mathbb{Z} / 2$ must itself have a free $\mathbb{Z} / 2$-action, so this module is isomorphic to the bordism module of $\mathbb{Z} / 2$-manifolds with free $\mathbb{Z} / 2$-action.

Proposition 3.7. The bordism module of $\mathbb{Z} / 2$-manifolds with free $\mathbb{Z} / 2$-action is isomorphic to $\mathfrak{N}_{*}(B \mathbb{Z} / 2)$.

Proof. Consider the following diagram:

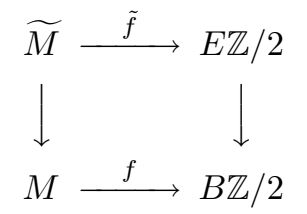


Given a representative $M$ with reference map $f$ to $B \mathbb{Z} / 2$, pull back the principal $\mathbb{Z} / 2$-bundle $E \mathbb{Z} / 2$ to get $\widetilde{M}$, which is in fact a free $\mathbb{Z} / 2$-manifold. Conversely, starting with a free $\mathbb{Z} / 2$-manifold $\widetilde{M}$, there is no obstruction to constructing a map $\tilde{f}$ to $E \mathbb{Z} / 2$. Pass to quotients to obtain $f: M \rightarrow B \mathbb{Z} / 2$.

These maps are well defined, as we apply the previous argument to the manifolds which act as bordisms. The composites of these maps are clearly identity maps.

Corollary 3.8. The module $\widetilde{\mathfrak{N}^{\mathbb{Z} / 2}}{ }_{*}(E \mathbb{Z} / 2+)$ is isomorphic to $\mathfrak{N}_{*}(B \mathbb{Z} / 2)$.

We may now deduce Theorem 3.1 We now give more geometric definitions of the maps in this exact sequence. We omit the proofs that these definitions coincide, which are straightforward.

Definition 3.9. Let $\eta: \mathfrak{N}_{*}(B \mathbb{Z} / 2) \rightarrow \mathfrak{N}_{*}^{\mathbb{Z} / 2}$ denote the $\mathfrak{N}_{*}$-module homomorphism which, using the identification of Proposition 3.7, sends a free $\mathbb{Z} / 2$-bordism class to the corresponding $\mathbb{Z} / 2$-bordism class.

Proposition 3.10. The homomorphism $\eta$ coincides with the homomorphism $i_{*}$ of the exact sequence (1) under the isomorphism of Corollary [3.8.

Definition 3.11. Let $\varphi: \mathfrak{N}_{*}^{\mathbb{Z} / 2} \rightarrow \bigoplus_{k} \mathfrak{N}_{*-k}(B O(k))$ be the map which sends a class $[M]$ to the fixed set of $[M]$ with reference map to $\sqcup B O(k)$ classifying the normal bundle to the fixed set.

Proposition 3.12. The homomorphism $\varphi$ coincides with the homomorphism $j_{*}$ of the exact sequence (10) under the isomorphism of Proposition 3.6.

Finally, we identify the boundary map.

Definition 3.13. Let $\delta: \bigoplus_{k} \mathfrak{N}_{*-k}(B O(k)) \rightarrow \mathfrak{N}_{*-1}(B \mathbb{Z} / 2)$ be the map of $\mathfrak{N}_{*^{-}}$ modules which sends $E$, a vector bundle, to the sphere bundle of $E$ with fiberwise $\mathbb{Z} / 2$-action defined by letting the non-trivial element of $\mathbb{Z} / 2$ act by multiplication by -1 .

Proposition 3.14. The homomorphism $\delta$ coincides with the homomorphism $\partial$ of the exact sequence of equation (1) under the isomorphisms of Proposition 3.6 and Corollary 3.8 .

Using theorems of Thom [11, we can identify $\mathfrak{N}_{*}(B \mathbb{Z} / 2)$ and $\bigoplus_{k} \mathfrak{N}_{*-k}(B O(k))$ given the standard computations of the mod 2 homology of $B \mathbb{Z} / 2$ and $B O(k)$.

Proposition 3.15. $\mathfrak{N}_{*}(B \mathbb{Z} / 2)$ is a free $\mathfrak{N}_{*}$-module generated by classes $x_{i}$ in degree $i$, where $i$ ranges over natural numbers. As the bordism module of free $\mathbb{Z} / 2$ manifolds, the generator $x_{i}$ is represented by the $i$-sphere with antipodal $\mathbb{Z} / 2$-action.

Proof. The mod 2 homology of $B \mathbb{Z} / 2=\mathbb{R} \mathbb{P}^{\infty}$ is well known to be $\mathbb{Z} / 2$ in every positive dimension. The class in dimension $i$ is the image of the fundamental class of $\mathbb{R} \mathbb{P}^{i}$ under inclusion. Under the identifications of Proposition 3.7 these classes correspond to spheres with antipodal action.

Next note that there are classifying maps for direct sums of the associated universal bundles $B O(k) \times B O(l) \rightarrow B O(k+l)$. These maps give rise to an $H$-space structure on $\bigsqcup B O(k)$, which in turn gives rise to a multiplication on homology. 
Proposition 3.16. As a ring, $\bigoplus_{k} \mathfrak{N}_{*-k}(B O(k))$ is a polynomial algebra over $\mathfrak{N}_{*}$ generated by classes $b_{i} \in \mathfrak{N}_{i-1}(B O(1))$ represented by the tautological line bundle over $\mathbb{R} \mathbb{P}^{i-1}$.

The fact that this ring is a polynomial algebra follows from the mod 2 homology computation, which is standard [9]. The fact that generators are represented by projective spaces is a straightforward Stiefel-Whitney number computation.

3.2. The tom Dieck localization sequence. Tom Dieck realized that there was a connection between the Conner-Floyd exact sequence and the localization methods in the equivariant $K$-theory of Atiyah and Segal. This connection has been fundamentally important in our work.

The following lemma provides translation between localization and topology. Once again, let $S$ be the multiplicative subset of $M O_{*}^{\mathbb{Z} / 2}$ generated by $e_{\sigma}$.

Lemma 3.17. As rings, $\widetilde{M O_{*}^{\mathbb{Z} / 2}}\left(S^{\oplus \infty \sigma}\right) \cong S^{-1} M O_{*}^{\mathbb{Z} / 2}$.

Proof. Apply $\widetilde{M O_{*}^{\mathbb{Z} / 2}}$ to the identification $S^{\oplus_{\infty} \sigma}=\underline{\lim } S^{\oplus_{n} \sigma}$. After applying the suspension isomorphisms $\widetilde{M O_{*}^{\mathbb{Z} / 2}}\left(S^{\oplus_{k} \sigma}\right) \cong \widetilde{M O_{*+1}^{\mathbb{Z} / 2}}\left(S^{\oplus_{k+1} \sigma}\right)$, the maps in the resulting directed system are multiplication by the $e_{\sigma}$.

Consider the cofiber sequence $S(\infty \sigma)_{+} \rightarrow S^{0} \rightarrow S^{\infty \sigma}$, which is a model for the sequence $E \mathbb{Z} / 2+\rightarrow S^{0} \rightarrow \widetilde{E \mathbb{Z} / 2}$, essentially our sequence of a pair from the previous section. By the previous lemma, after applying $\widehat{M O^{\mathbb{Z}} / 2} *$ to this cofiber sequence, the second map in this sequence is the canonical map from $M O_{*}^{\mathbb{Z} / 2}$ to $S^{-1} M O_{*}^{\mathbb{Z} / 2}$. We now identify the outside terms in this sequence.

Theorem 3.18. $\widetilde{M O^{\mathbb{Z} / 2}}{ }_{*}(\widetilde{E \mathbb{Z} / 2}) \cong \bigoplus_{k \in \mathbb{Z}} \mathfrak{N}_{*-k}(B O)$.

Proof. Recall the definition of $M O_{*}^{\mathbb{Z} / 2}(\widetilde{E \mathbb{Z} / 2})$ and consider the space of maps from $S^{V}$ to $\widetilde{E \mathbb{Z} / 2} \wedge T\left(\xi_{n}^{\mathbb{Z} / 2}\right)$, for any representation $V$.

First we show that for any $\mathbb{Z} / 2$-spaces $X$ and $Y$, the restriction map

$$
\operatorname{Maps}^{\mathbb{Z} / 2}(X, \widetilde{E \mathbb{Z} / 2} \wedge Y) \rightarrow \operatorname{Maps}\left(X^{\mathbb{Z} / 2},(\widetilde{E \mathbb{Z} / 2} \wedge Y)^{\mathbb{Z} / 2}\right)=\operatorname{Maps}\left(X^{\mathbb{Z} / 2}, Y^{\mathbb{Z} / 2}\right)
$$

is an equivalence. First note that this restriction of mapping spaces is a fibration. Over a given component of $\operatorname{Maps}\left(X^{\mathbb{Z} / 2}, Y^{\mathbb{Z} / 2}\right)$ a fiber is going to be the space of maps from $X$ to $\widetilde{E \mathbb{Z} / 2} \wedge Y$ which are specified on $X^{\mathbb{Z} / 2}$. We filter this mapping space by filtering $X$. Because the maps are already specified on $X^{\mathbb{Z} / 2}$, we need only adjoin cells of the form $G \times D^{n}$, where $G$ denotes $\mathbb{Z} / 2$ acting on itself by left multiplication. Hence the subquotients in this filtration will be spaces of equivariant maps from $\mathbb{Z} / 2 \times D^{n}$ to $\widetilde{E \mathbb{Z} / 2} \wedge Y$ whose restriction to the boundary of $\mathbb{Z} / 2 \times D^{n}$ is specified. Because $\mathbb{Z} / 2 \times D^{n}$ is a free $\mathbb{Z} / 2$-space, it suffices to consider the restriction of such a map to one copy of $D^{n}$. But $\widetilde{E Z / 2} \wedge Y$ is contractible, hence so is this mapping space. Therefore the fibers of our restriction map are contractible.

Applying this argument for $X=S^{V}, Y=T\left(\xi_{n}^{\mathbb{Z} / 2}\right)$ we see that our computation follows from knowledge of $T\left(\xi_{n}^{\mathbb{Z} / 2}\right)^{\mathbb{Z} / 2}$. We claim that $T\left(\xi_{n}^{\mathbb{Z} / 2}\right)^{\mathbb{Z} / 2}=\bigvee T\left(\xi_{i}\right) \wedge$ $B O(n-i)$. We show this by analysis of the fixed set of $\xi_{n}^{\mathbb{Z} / 2}$. Any fixed point 
of $\xi_{n}^{\mathbb{Z} / 2}$ must lie over a fixed point of $B O^{\mathbb{Z} / 2}(n)$. The fixed set of $B O^{\mathbb{Z} / 2}(n)$ is the classifying space for $\mathbb{Z} / 2$-vector bundles over trivial $\mathbb{Z} / 2$-spaces. A vector bundle over a trivial $\mathbb{Z} / 2$-space decomposes as a direct sum according to decomposition of fibers according to representation type. As there are only two representation types for $\mathbb{Z} / 2$, we deduce that $\left(B O^{\mathbb{Z} / 2}(n)\right)^{\mathbb{Z} / 2}=\bigsqcup B O(i) \times B O(n-i)$. Restricted to a component of this fixed set $\xi_{n}^{\mathbb{Z} / 2}$ will be $\xi(i) \times \xi(n-i)$ where $\mathbb{Z} / 2$ fixes all points in the first factor and acts by multiplication by -1 on fibers in the second factor. Hence one component of the fixed set of $\xi_{n}^{\mathbb{Z} / 2}$ will be $\xi(i) \times B O(n-i)$.

Passing to Thom spaces we find

$$
T\left(\xi_{n}^{\mathbb{Z} / 2}\right)^{\mathbb{Z} / 2}=\bigvee T(\xi(i)) \wedge B O(n-i)_{+} .
$$

Using this result along with our first reduction we see that

$$
\left[S^{V}, S^{\infty \sigma} \wedge T\left(\xi_{n}^{\mathbb{Z} / 2}\right)\right]^{\mathbb{Z} / 2}=\left[S^{V^{\mathbb{Z} / 2}}, \bigvee T(\xi(i)) \wedge B O(n-i)_{+}\right]
$$

The theorem follows by passing to direct limits.

As $\widetilde{M O^{\mathbb{Z} / 2}}{ }_{*}(\widetilde{E \mathbb{Z} / 2}) \cong S^{-1} M O_{*}^{\mathbb{Z} / 2}$ we are interested in multiplicative structures as well.

Corollary 3.19. $S^{-1} M O_{*}^{\mathbb{Z} / 2} \cong \mathfrak{N}_{*}\left[x_{i}, e, e^{-1}\right]$, where as elements of $\bigoplus_{k} \mathfrak{N}_{*-k}(B O)$, $x_{i}$ are the images of the generators given in Proposition 3.16 under the canonical inclusions of $B O(k)$ into $B O$, and as an element of $S^{-1} M O_{*}^{\mathbb{Z} / 2}$, $e$ is the image of the Euler class $e_{\sigma}$.

Proof. The multiplication on $M O_{*}^{\mathbb{Z} / 2}$ restricts to $T\left(\xi_{j}^{\mathbb{Z} / 2}\right)^{\mathbb{Z} / 2}=\bigvee T(\xi(i)) \wedge$ $B O(n-i)_{+}$as the standard multiplication on $T(\xi(i))$ factors smashed with the classifying map for the Whitney sum on $B O(n-i)_{+}$factors.

Passing to the direct limit and neglecting grading, we are computing $\mathfrak{N}_{*}(\mathbb{Z} \times B O)$, where $\mathbb{Z} \times B O$ has an $H$-space structure which is the product of the group structure on $\mathbb{Z}$ and the $H$-space structure on $B O$ arising from Whitney sum. Let $e^{-1}$ denote the unit class in $\mathfrak{N}_{*}(B O \times 1)$ in degree 1 . The result follows by noting that $\mathfrak{N}_{*}(B O)$ is a polynomial ring in classes $x_{i}$ where $x_{i}$ is the image of the generator of $\mathfrak{N}_{i}(B O(1))$ under the inclusion from $B O(1)$ to $B O$. That $e$ is the image of $e_{\sigma}$ follows directly from their definitions, chasing through the identifications of Theorem 3.18 ,

Theorem 3.20. $\widetilde{M O^{\mathbb{Z} / 2}} *(E \mathbb{Z} / 2+) \cong \mathfrak{N}_{*}(B \mathbb{Z} / 2)$.

Proof. In the spirit of giving elementary proofs, we argue geometrically as follows. Consider a $\mathbb{Z} / 2$-map from $S^{V}$ to $E \mathbb{Z} / 2+\bigwedge T\left(\xi_{n}^{\mathbb{Z} / 2}\right)$. Because the latter space has a free $\mathbb{Z} / 2$-action away from the basepoint, $\left(S^{V}\right)^{\mathbb{Z} / 2}$ must map to the basepoint. If we pass to the map from the quotient $S^{V} /\left(S^{V}\right)^{\mathbb{Z} / 2}$, we have a $\mathbb{Z} / 2$-map between $\mathbb{Z} / 2$-spaces which are free $\mathbb{Z} / 2$-manifolds away from their basepoints. We now use transversality arguments, which hold in the presence of free $\mathbb{Z} / 2$-actions. Given a $\mathbb{Z} / 2$-map from $S^{V} /\left(S^{V}\right)^{\mathbb{Z} / 2}$ to $E \mathbb{Z} / 2+\bigwedge T\left(\xi_{n}^{\mathbb{Z} / 2}\right)$ we may homotop it locally to a map which is transverse regular to the zero section of $T\left(\xi_{n}^{\mathbb{Z} / 2}\right)$ and pull back a sub-manifold of $S^{V} /\left(S^{V}\right)^{\mathbb{Z} / 2}$ which must necessarily be free.

Hence, we identify $\widetilde{M O^{\mathbb{Z} / 2}}{ }_{*}(E \mathbb{Z} / 2+)$ with the bordism module of free $\mathbb{Z} / 2$ manifolds. The theorem follows from Proposition 3.7 
As one should suspect at this point, the tom Dieck localization sequence is precisely the homotopical version of the Conner-Floyd sequence. The following theorem is due to tom Dieck in the complex setting.

Theorem 3.21. The diagram

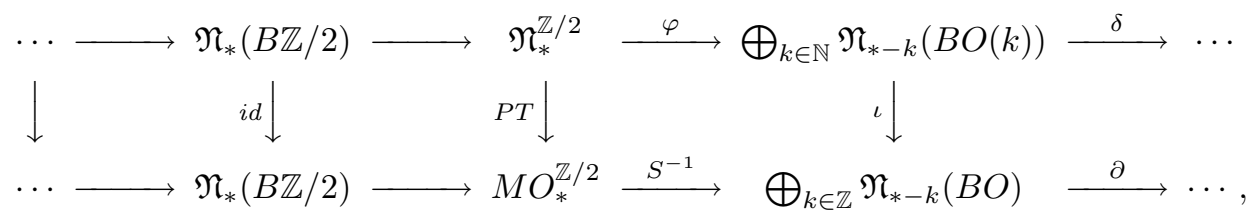

where the first vertical map is the identity map, the second is the Pontryagin-Thom map and the third is defined by the standard inclusion of $B O(k)$ into $B O$, commutes.

\subsection{Proofs of the main results.}

Proof of Theorem 2.1. We show that the images of $e_{\sigma}$ and $[\mathbb{P}(n \tau \oplus \sigma)]$ along with $e_{\sigma}^{-1}$ generate $S^{-1} M O_{*}^{\mathbb{Z} / 2}$.

Consider the following diagram:

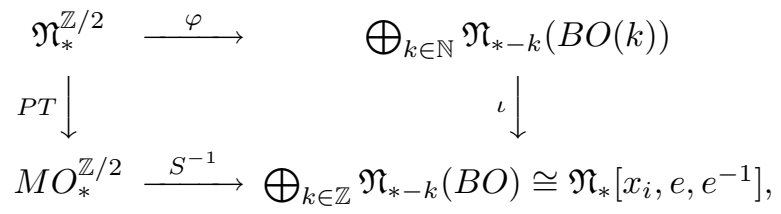

which combines results of Theorem 3.21 and Corollary 3.19 To compute the image of $[\mathbb{P}(n \tau \oplus \sigma)]$ under localization it suffices to look at fixed-set data, because it is a geometric class.

There are two components of the fixed set $[\mathbb{P}(n \tau \oplus \sigma)]^{\mathbb{Z} / 2}$. Using homogeneous coordinates $\left[y_{0}, \ldots, y_{n}\right]$, these components are defined by the conditions $y_{n}=0$ and $y_{1}=\cdots=y_{n-1}=0$. The condition $y_{n}=0$ defines an $(n-1)$-dimensional projective space. Its normal bundle is the tautological line bundle. The condition $y_{1}=\cdots=y_{n-1}=0$ defines an isolated fixed point which has an $n$-dimensional normal bundle. Using the generators named in Corollary 3.19 we have

$$
S^{-1}[\mathbb{P}(n \tau \oplus \sigma)]=x_{n}+e^{-(n+1)} .
$$

In Corollary 3.19 we also noted that the image of $e_{\sigma}$ under localization was $e$. It thus follows that the images of $e_{\sigma}$ and $[\mathbb{P}(n \tau \oplus \sigma)]$ under localization, along with $e_{\sigma}^{-1}$, generate $S^{-1} M O_{*}^{\mathbb{Z} / 2} \cong \mathfrak{N}_{*}\left[x_{i}, e, e^{-1}\right]$, which is what was to be shown.

Proof of Theorem 2.2. The exact sequence in question is a Gysin sequence. Apply $\widetilde{M O_{\mathbb{Z} / 2}}$ to the cofiber sequence $G_{+} \stackrel{i}{\rightarrow} S^{0} \stackrel{j}{\rightarrow} S^{\sigma}$, where the first map is projection of $G$ onto the non-basepoint of $S^{0}$. The resulting long exact sequence is

$$
\cdots \rightarrow{\widetilde{M O_{\mathbb{Z} / 2}}}^{n}\left(S^{\sigma}\right) \stackrel{j^{*}}{\rightarrow} M O_{\mathbb{Z} / 2}{ }^{n} \stackrel{i^{*}}{\rightarrow}{\widetilde{M O_{\mathbb{Z} / 2}}}^{*}\left(G_{+}\right) \stackrel{\delta}{\rightarrow}{\widetilde{M O_{\mathbb{Z} / 2}}}^{n+1}\left(S^{\sigma}\right) \rightarrow \cdots
$$

By the periodicity of $M O^{\mathbb{Z} / 2},{\widetilde{M O_{\mathbb{Z} / 2}}}^{n}\left(S^{\sigma}\right) \cong M O_{\mathbb{Z} / 2}{ }^{n-2}$. By definition $j^{*}$ is multiplication by $e_{\sigma}$. From the fact that $\operatorname{Maps}^{\mathbb{Z} / 2}\left[G_{+}, Y\right]$ is homeomorphic to $Y$ for any $\mathbb{Z} / 2$-space $Y$, we see that $\widehat{M O_{\mathbb{Z} / 2}}{ }^{*}\left(G_{+}\right) \cong \mathfrak{N}_{*}$ and $i^{*}$ is the augmentation $\operatorname{map} \alpha$. 
As we remarked after the statement of Theorem [2.2, the augmentation map $\alpha$ is split. Hence our long exact sequence breaks up into short exact sequences, and the result follows.

Proof of Theorem 2.4. First we verify relations. Then we show that the classes listed generate $M O_{*}^{\mathbb{Z} / 2}$. Finally we show that the relations are a complete set of relations. We may view $M O_{*}^{\mathbb{Z} / 2}$ as a subring of $S^{-1} M O_{*}^{\mathbb{Z} / 2} \cong \mathfrak{N}_{*}\left[x_{i}, e, e^{-1}\right]$, which we can do as $e_{\sigma}$ is not a zero divisor. Hence, we may verify the second family of relations by direct computation. The first family of relations holds by definition.

For convenience, rename $[\mathbb{P}(n \tau \oplus \sigma)]$ as $X_{n}$. By Theorem 2.1 , any class in $M O_{*}^{\mathbb{Z} / 2}$ when multiplied by some power of $e_{\sigma}$ is equal to a class in $R_{*}$ modulo the annihilator ideal of $e_{\sigma}$, which is zero. Hence we may filter $M O_{*}^{\mathbb{Z} / 2}$ exhaustively as

$$
R_{*}=R_{*}^{0} \subset R_{*}^{1} \subset \cdots \subset M O_{*}^{\mathbb{Z} / 2}
$$

where $R_{*}^{i}$ is obtained by adjoining to $R_{*}^{i-1}$ all $x \in M O_{*}^{\mathbb{Z} / 2}$ such that $x \cdot e_{\sigma}=y \in$ $R_{*}^{i-1}$. By Theorem 2.2 the set of all such $y$ is $\operatorname{Ker}(\alpha) \cap R_{*}^{i-1}$. The kernel of the augmentation map is clearly generated by all classes $y-\bar{y}$. So we may obtain $R_{*}^{i}$ from $R_{*}^{i-1}$ by applying $\Gamma$ to every class in $R_{*}^{i-1}$. Since $\Gamma(x y)=\Gamma(x) y-\bar{x} \Gamma(y)$, it suffices to apply $\Gamma$ only to primitive elements. It follows that $e_{\sigma}$ and $\Gamma^{i}\left(X_{n}\right)$ constitute multiplicative generators.

Finally, to show these relations are complete we identify an additive basis of $M O_{*}^{\mathbb{Z} / 2}$. There are two types of monomials in the additive basis, those of the form $e_{\sigma}^{k} f, f \in \mathfrak{N}_{*}\left[X_{i}\right]$, and those of the form $\Gamma^{k}\left(X_{j}\right) f, f \in \mathfrak{N}_{*}\left[X_{i} \mid i>j\right]$. We may check that these classes are additively independent by mapping to $S^{-1} M O_{*}^{\mathbb{Z} / 2}$. Define the complication of a monomial in our basis elements to be the sum of the number of times both $e_{\sigma}$ and $\Gamma$ appear in the monomial and the sum of all $i$ where $\Gamma^{i}\left(X_{k}\right)$ appears for some $X_{k}$ where $k$ is not minimal among the $X_{i}$ which appear. And define the complication of a sum of monomials to be the greatest of their individual complications. We may use our two families of relations to decrease complication, which inductively allows us to reduce to our additive basis whose members have zero complication.

Corollary 3.22. An additive basis for $M O_{*}^{\mathbb{Z} / 2}$ is given by monomials of the form $e_{\sigma}^{k} f, f \in \mathfrak{N}_{*}\left[X_{i}\right]$, and those of the form $\Gamma^{k}\left(X_{j}\right) f, f \in \mathfrak{N}_{*}\left[X_{i} \mid i>j\right]$.

Proof of Theorem 2.6. Once again we use the fact that the map from $M O_{*}^{\mathbb{Z} / 2}$ to $S^{-1} M O_{*}^{\mathbb{Z} / 2} \cong \mathfrak{N}_{*}\left[x_{i}, e, e^{-1}\right]$ is a faithful representation, along with direct computation. By Theorem 3.21 , we may compute the image of $[\gamma(M)]$ under localization by analyzing fixed-set data.

Recalling the definition of $\gamma(M)$ we see two types of fixed points $[m, x, y]$ under the $\mathbb{Z} / 2$-action, those with $x=0$ and those with $y=0$ and $g m=m$. The first fixed set is $\alpha(M)$, with a trivial normal bundle. The second fixed set is the fixed set of $M$, whose normal bundle is the normal bundle of this fixed set in $M$ crossed with a trivial bundle. Because multiplication by $e^{-1}$ in $S^{-1} M O_{*}^{\mathbb{Z} / 2}$ corresponds geometrically to crossing with a trivial bundle, this fixed set is the fixed set of $\Gamma([M])$.

Proof of Theorem 2.7. By analysis identical to that in the proof of Theorem 2.2 the map $\varphi: \mathfrak{N}_{*}^{\mathbb{Z} / 2} \rightarrow \bigoplus_{k} \mathfrak{N}_{*-k}(B O(k))$ is injective. 
We deduce from the comparison of exact sequences in Theorem 3.21 that the Pontryagin-Thom map from $\mathfrak{N}_{*}^{\mathbb{Z} / 2}$ to $M O_{*}^{\mathbb{Z} / 2}$ is injective. So the image of $\varphi$ is the image of $M O_{*}^{\mathbb{Z} / 2}$ in the subring $\mathfrak{N}_{*}\left[x_{i}, e^{-1}\right]$ of $S^{-1} M O_{*}^{\mathbb{Z} / 2}$. The images of $\left[\gamma^{i}(\mathbb{P}(n \tau \oplus \sigma))\right]$ generate this image, so these classes generate $\mathfrak{N}_{*}^{\mathbb{Z} / 2}$.

Proof of Theorem 2.8. This theorem follows from Corollary 3.22 and Theorem 2.7 Any monomial of the form $\Gamma^{k}([\mathbb{P}(j \tau \oplus \sigma)]) f$, where $f \in \mathfrak{N}_{*}[\mathbb{P}(i \tau \oplus \sigma)]$ for $i>j$, is in fact in the image of the Pontryagin-Thom map. Monomials of the form $e_{\sigma}^{k} f$, $f \in \mathfrak{N}_{*}[\mathbb{P}(i \tau \oplus \sigma)]$, are generated over $\mathfrak{N}_{*}^{\mathbb{Z} / 2}$ by $e_{\sigma}^{k}$ which we denote by $x_{k}$. The module relations for the quotient follow from the ring relations for $M O_{*}^{\mathbb{Z} / 2}$.

\section{REFERENCES}

[1] J. C. Alexander. The Bordism Ring of Manifolds with Involution. Proceedings of the A.M.S. 31 (1972), 536-542. MR 44:7563

[2] T. Bröcker and E. Hook. Stable Equivariant Bordism. Mathematische Zeitschrift 129 (1972), 269-277. MR 47:9652

[3] G. Carlsson. A Survey of Equivariant Stable Homotopy Theory. Topology 31 (1992), 1-27. MR 93d:55009

[4] P. E. Conner and E. E. Floyd. Differentiable Periodic Maps. Springer, Berlin-Heidelberg-New York, 1964. MR 31:750

[5] S. R. Costenoble. The structure of some equivariant Thom spectra. Transactions of the A.M.S. 315 (1989), 231-254. MR 89m:57038

[6] S. R. Costenoble and S. Waner. G-transversality revisited. Proceedings of the A.M.S. 116 (1992), 535-546. MR 92m:57044

[7] T. tom Dieck. Bordism of G-Manifolds and Integrality Theorems. Topology 9 (1970), 345-358. MR 42:1148

[8] J.P. May, et al. Equivariant Homotopy and Cohomology Theory. Volume 91 of the CBMS Regional Conference Series in Mathematics. AMS Publications, Providence, 1996. MR 97k:55016

[9] J. Milnor and J. Stasheff. Characteristic Classes. Volume 76 of Annals of Mathematics Studies. Princeton University Press, Princeton, 1974. MR 55:13428

[10] D. P. Sinha. Computations in Complex Equivariant Bordism Theory. Preprint, 1999.

[11] R. Thom. Sur quelques proprétés globales des varietés differentiables. Commentarii Mathematici Helvetici 28 (1954), 17-86. MR 15:890a

Department of Mathematics, Brown University, Providence, Rhode Island 02906

E-mail address: dps@math.brown.edu 